\title{
Roepke, Martina, Privat-Vorstellung. Heimkino in Deutschland
}

\section{Christian Joschke}

\section{(2) OpenEdition}

Journals

Édition électronique

URL : http://journals.openedition.org/ifha/1724

DOI : $10.4000 /$ ifha. 1724

ISSN : 2198-8943

\section{Éditeur}

IFRA - Institut franco-allemand (sciences historiques et sociales)

Référence électronique

Christian Joschke, "Roepke, Martina, Privat-Vorstellung. Heimkino in Deutschland», Revue de l'IFHA [En ligne], Date de recension, mis en ligne le 01 janvier 2008, consulté le 22 septembre 2020. URL : http:// journals.openedition.org/ifha/1724; DOI : https://doi.org/10.4000/ifha.1724

Ce document a été généré automatiquement le 22 septembre 2020.

(CIFHA 


\title{
Roepke, Martina, Privat-Vorstellung. Heimkino in Deutschland
}

\author{
Christian Joschke
}

La découverte progressive, ces dernières années, d'un patrimoine considérable mais longtemps négligé de photographies ou de films d'amateurs suscite aujourd'hui l'intérêt légitime de l'historien soucieux de donner une place aux acteurs historiques les plus modestes et de reprendre ainsi à son compte les méthodes de l'histoire du quotidien. L'étude de M.R. s'inscrit dans ce champ en étudiant des films privés produits en Allemagne avant 1945, et en particulier un fonds de films amateurs de la période national-socialiste, réunis à la fin des années soixante-dix par une équipe de la télévision Süddeutscher Rundfunk (SDR), aujourd'hui Südwestrundfunk (SWR, Stuttgart). On comprend d'emblée toute la portée que peut et doit avoir une telle étude sur le rapport des classes moyennes à la culture et à l'idéologie dominante dans le contexte nazi. Mais cette étude, nous dit M.R., n'a pas l'ambition de répondre à la question galvaudée de la banalité du fascisme ; elle s'attache à reconstruire l'espace des possibles ouvert grâce à la pratique amateur à l'intérieur des cadres normatifs imposés par la projection privée et par la distribution des rôles sociaux dans la vie quotidienne des années trente.

La fidélité aux méthodes déployées par l'histoire du quotidien explique d'abord l'élargissement fort judicieux du cadre d'analyse : l'abolition de la frontière entre film « documentaire " privé et fiction d'amateurs ; l'élargissement de la notion de cinéma privé aux films produits et distribués par les entreprises productrices de matériel ; la prise en compte des méthodes de la sociologie communicationnelle, qui incite l'auteure à étudier les dispositifs de la prise de vue et de la projection.

Ces méthodes justifient également le choix de confronter les sources imprimées, qui prescrivent les normes culturelles, aux films produits en amateur. Le plan de l'ouvrage en est particulièrement révélateur. Après une introduction riche en considérations méthodologiques, M.R. trace l'histoire du film amateur depuis la commercialisation du film 17,5 mm en 1903 par Ernemann jusqu'à l'introduction en Allemagne, en 1925, du film $16 \mathrm{~mm}$, qui devient, à côté du $8 \mathrm{~mm}$, le standard international du film amateur. Elle 
évoque aussi précisément comment se constituent progressivement un savoir et un discours véhiculés par divers supports, et comment s'organise la distribution des films : le rôle d'Ernemann avant la Première Guerre mondiale, puis celui du Bund deutscher Film Amateure (BDFA) après 1927. On comprend mieux, ainsi, avec quelle facilité le régime national-socialiste a pris en main un réseau non politisé, dominé par les lobbies industriels : on assiste à la mise au pas du BDFA, à l'application de la loi du 16 février 1934 (Reichslichtspielgesetz), à l'introduction de la propagande par le biais des Kulturfilme, enfin, à la constitution, en 1937, d'un réseau de distribution spécifique, le Zentraler Schmalfilmverleih, dont les liens avec le Ministère de la Propagande mériteraient d'être mieux explicités.

Dans le deuxième chapitre, M.R. développe grâce aux méthodes de la sociologie de la communication une étude des dispositifs, des rôles sociaux, des figures cinématographiques (le clown, le boycotteur, le coquet, l'assistant, le complice), des espaces et des temps de la projection. Ce canevas sert de base au troisième chapitre, qui se penche avec minutie sur quelques productions des années trente et révèle l'espace des possibles ouvert par la pratique amateur. La pratique du film permet la distanciation par rapport aux rôles sociaux comme le montre l'analyse du film Grosse Ereignisse werfen ihre Schatten voraus $(8 \mathrm{~mm}, 1937)$, ou encore l'intégration dans le montage de morceaux de films commerciaux censés représenter, avec par exemple une évocation du cabaret, le rêve licencieux du personnage principal dans Eine Nacht und ein Morgen (8 mm, 1937).

Si les bénéfices tirés des méthodes de l'« histoire du quotidien ", de celles de la sociologie et d'une perspective élargie aux dispositifs de prise de vue et de projection sont indéniables, on regrettera néanmoins que la construction de l'ouvrage ne permette guère à M.R. de revenir au contexte historique général après l'évocation des cas particuliers analysés dans le troisième chapitre. La conclusion reste donc relativement pauvre : le cinéma amateur y apparaît comme le document d'une " pratique médiale ", il " n'est pas le miroir de la réalité du Troisième Reich, mais simplement une partie » (p. 212). On décèlera, dans le choix d'un parcours à sens unique allant du général au particulier, un indéniable effet de source. Malgré les nombreuses précautions méthodologiques reprises avec justesse à Roger Odin, malgré les entretiens qu'elle a menés avec des témoins de situations variées, M.R. ne parvient guère à mobiliser les informations nécessaires pour replacer les films dans leur contexte historique particulier : quels sont les situations sociales des familles, leurs lieux d'habitation, leur histoire ? Les bobines du SWR ne répondent pas à ces questions et la trame théorique déployée ici pour imaginer les situations communicationnelles des films ne suffit pas à combler cette lacune.

Christian Joschke (Université Lumière - Lyon II) 